Arch. Math. 92 (2009), 129-136

(C) 2009 Birkhäuser Verlag Basel/Switzerland

0003/889X/020129-8, published online 2009-02-18

DOI $10.1007 / \mathrm{s} 00013-008-2925-0$

Archiv der Mathematik

\title{
Normal forms for real quadratic forms
}

\author{
Bernhard KrÖtz And HenRIK SchlichtKRUll
}

\begin{abstract}
We investigate the non-diagonal normal forms of a quadratic form on $\mathbb{R}^{n}$, in particular for $n=3$. For this case it is shown that the set of normal forms is the closure of a 5 -dimensional submanifold in the 6 -dimensional Grassmannian of 2-dimensional subspaces of $\mathbb{R}^{5}$.
\end{abstract}

Mathematics Subject Classification (2000). 11E10, 17B20.

Keywords. Real quadratic form, diagonalization, semisimple Lie algebra.

1. Introduction. According to the principal axes theorem every real quadratic form in $n$ variables allows an orthogonal diagonalization with normal form

$$
A_{1} x_{1}^{2}+\cdots+A_{n} x_{n}^{2}
$$

where $A_{1}, \ldots, A_{n} \in \mathbb{R}$. In this article we investigate (for the case $n=3$ ) the existence of other normal forms.

To be more precise, let $q_{1}, \ldots, q_{n}$ be quadratic forms on $\mathbb{R}^{n}$. If for every quadratic form $q$ on $n$-dimensional Euclidean space there exists an orthonormal basis in which $q$ takes the form

$$
q(x)=A_{1} q_{1}(x)+\cdots+A_{n} q_{n}(x)
$$

for some set of coefficients, we say that this expression is a normal form of $q$.

Passing to matrices, let us consider $V=\operatorname{Sym}(n, \mathbb{R})$, the vector space of symmetric $n \times n$-matrices. On $V$ there is the natural action of the special orthogonal group $K:=S O(n, \mathbb{R})$ by conjugation, say

$$
k \cdot X:=k X k^{-1} \quad(k \in K, X \in V) .
$$

If $D$ denotes the space of diagonal matrices in $V$, then the principal axes theorem asserts that

$$
V=K \cdot D:=\{k \cdot d \mid k \in K, d \in D\}
$$


Furthermore if $d_{1}, d_{2} \in D$, then $K \cdot d_{2}=K \cdot d_{1}$ if and only if $d_{2}$ is obtained from $d_{1}$ by a permutation of coordinates (the set of eigenvalues is unique).

The question we address is, for which n-dimensional subspaces $W$ in $V$ is $V=K \cdot W$ ? It would be tempting to assert that the unique property of $D$ (and its conjugates by $K$ ), which causes the principal axes theorem, is that it is abelian with respect to matrix multiplication. However this is not correct, in fact there exist non-abelian $n$-dimensional subspaces $W$ with $V=K \cdot W$ (see the example below).

There is some redundancy in the problem, namely the center of $V$ on which $K$ acts trivially. Let us remove that and define $\mathfrak{p}:=V_{\mathrm{tr}=0}$ to be the space of zerotrace elements in $V$. Likewise we set $\mathfrak{a}:=D_{\mathrm{tr}=0}$. The principal axes theorem now reads as

$$
\mathfrak{p}=K \cdot \mathfrak{a},
$$

and the problem we pose (and solve for $n=3$ ) is to determine the set of subspaces

$$
\mathcal{X}:=\{W \subset \mathfrak{p} \mid \operatorname{dim} W=2, K \cdot W=\mathfrak{p}\} .
$$

The following example shows that there are elements in $\mathcal{X}$ which are not conjugate to $\mathfrak{a}$.

Example 1.1. Let $\mathfrak{p}=\operatorname{Sym}(3, \mathbb{R})_{\operatorname{tr}=0}$ and $K=\operatorname{SO}(3, \mathbb{R})$. Define

$$
W:=\left\{X_{\mu \lambda}:=\left(\begin{array}{ccc}
\mu & 0 & \lambda \\
0 & -\mu & 0 \\
\lambda & 0 & 0
\end{array}\right) \mid \mu, \lambda \in \mathbb{R}\right\} .
$$

Then $K \cdot W=\mathfrak{p}$, and $W$ is not abelian.

Proof. A more general result will be established later. Here we can give a simple proof.

Let $A \in \mathfrak{p}$ be given, and let $\nu_{1} \geq \nu_{2} \geq \nu_{3}$ be its eigenvalues. Then $\nu_{1}+\nu_{2}+\nu_{3}=$ 0 , and hence $\nu_{1} \geq 0 \geq \nu_{3}$. Let

$$
\mu=-\nu_{2}=\nu_{1}+\nu_{3} \quad, \quad \lambda=\sqrt{-\nu_{1} \nu_{3}} .
$$

The matrix $X_{\mu \lambda}$ has the characteristic polynomial

$$
\begin{aligned}
\operatorname{det}\left(\begin{array}{ccc}
\mu-x & 0 & \lambda \\
0 & -\mu-x & 0 \\
\lambda & 0 & -x
\end{array}\right) & =(-\mu-x)\left(-(\mu-x) x-\lambda^{2}\right) \\
& =\left(\nu_{2}-x\right)\left(x-\nu_{1}\right)\left(x-\nu_{3}\right)
\end{aligned}
$$

Hence $A$ and $X_{\mu \lambda}$ have the same eigenvalues, and thus they are conjugate. It is easily seen that $X_{10} X_{01} \neq X_{01} X_{10}$.

Corollary 1.2. Every trace free real quadratic form in three variables allows a normal form of the type

for $A, B \in \mathbb{R}$.

$$
A\left(x^{2}-y^{2}\right)+B x z
$$


The problem can be stated in a more general setting. Let us consider a real semi-simple Lie algebra $\mathfrak{g}$ with Cartan decomposition $\mathfrak{g}=\mathfrak{k}+\mathfrak{p}$. The space $\mathfrak{p}=$ $\operatorname{Sym}(n, \mathbb{R})_{\operatorname{tr}=0}$ is obtained in the special case $\mathfrak{g}=\mathfrak{s l}(n, \mathbb{R})$. Let $\mathfrak{a} \subset \mathfrak{p}$ be a maximal abelian subspace and $K=e^{\text {ad } \mathfrak{k}}$. According to standard structure theory of semisimple Lie algebras (see for example [1, Theorem 6.51]) the following generalization of the principal axes theorem holds:

- $\mathfrak{p}=K \cdot \mathfrak{a}$.

- $K \cdot X=K \cdot Y$ for $X, Y \in \mathfrak{a}$ if and only if $\mathcal{W} \cdot X=\mathcal{W} \cdot Y$ where $\mathcal{W}=$ $N_{K}(\mathfrak{a}) / Z_{K}(\mathfrak{a})$ is the Weyl group.

Let $r:=\operatorname{dim} \mathfrak{a}$ be the real rank of $\mathfrak{g}$. We consider $\operatorname{Gr}_{r}(\mathfrak{p})$ the Grassmannian of $r$-dimensional subspaces in $\mathfrak{p}$. Inside of $\operatorname{Gr}_{r}(\mathfrak{p})$ we consider the subset

$$
\mathcal{X}:=\left\{W \in \operatorname{Gr}_{r}(\mathfrak{p}) \mid K \cdot W=\mathfrak{p}\right\} .
$$

Let

$$
\mathcal{X}_{\mathrm{ab}}:=\left\{W \in \operatorname{Gr}_{r}(\mathfrak{p}) \mid W \text { abelian }\right\} .
$$

Then the following are immediate (by the same reference as before):

- $\mathcal{X} \supset \mathcal{X}_{\mathrm{ab}} \simeq K / N$ where $N=N_{K}(\mathfrak{a})$.

- If $r=1$ then $\mathcal{X}=\mathcal{X}_{\mathrm{ab}}=\mathrm{Gr}_{r}(\mathfrak{p})$.

If $r \geq 2$ and $\mathfrak{g}$ simple, then $\mathcal{X} \subsetneq \operatorname{Gr}_{r}(\mathfrak{p})$, and we expect $\mathcal{X}_{\mathrm{ab}} \subsetneq \mathcal{X}$, as in Example 1.1 .

Here we describe the set $\mathcal{X}$ for $\mathfrak{g}=\mathfrak{s l}(3, \mathbb{R})$, in which case $r=2, \operatorname{dim} \operatorname{Gr}_{2}(\mathfrak{p})=6$ and $\operatorname{dim} \mathcal{X}_{\mathrm{ab}}=2$. It turns out that $\mathcal{X}$ is dominated by a real algebraic variety of dimension 5: There exists a surjective algebraic map

$$
\Phi: K \times_{N_{0}} \mathbb{P}\left(\mathbb{R}^{3}\right) \rightarrow \mathcal{X}
$$

with $N_{0} \simeq(\mathbb{Z} / 4 \mathbb{Z}) \rtimes \mathbb{Z} / 2 \mathbb{Z}$ and generically trivial fibers (see Theorem 3.2 in Section 3 below)

It would be of interest to obtain a similar description for the general case. In particular this would provide alternative parametrizations of the $K$-orbits in $\mathfrak{p}$. In an extended version of this article (see arXiv:0805.0180), we describe an approach by tools from algebraic geometry, which could possibly be of use in such an investigation.

2. Description by invariants. Let $\mathfrak{g}=\mathfrak{s} l(3, \mathbb{R})$ and $\mathfrak{a}=\operatorname{diag}(3, \mathbb{R})_{\operatorname{tr}=0}$. We give the following description of $\mathcal{X}$, which will lead to the classification in the following section.

Proposition 2.1. The two dimensional subspace $W \in \mathrm{Gr}_{2}(\mathfrak{p})$ belongs to $\mathcal{X}$ if and only if it contains a non-zero matrix $X$ with two equal eigenvalues.

For example, with the notation in Example 1.1, the matrix $X_{\mu \lambda}$ with $\mu=-1$ and $\lambda=\sqrt{2}$ has eigenvalues $1,1,-2$. Hence the space $W$ in this example belongs to $\mathcal{X}$. 
Proof. That this is a necessary condition is clear, since $W \in \mathcal{X}$ means that $W$ contains at least one element from every $K$-orbit on $\mathfrak{p}$.

In order to describe the $K$-orbits, we recall some basic invariant theory. Let

$$
\begin{aligned}
& u_{1}(X)=\operatorname{tr} X^{2} \\
& u_{2}(X)=\operatorname{det} X
\end{aligned}
$$

for $X \in \mathfrak{p}$. Then $u_{1}, u_{2} \in \mathbb{C}[\mathfrak{p}]^{K}$, the ring of $K$-invariant polynomials on $\mathfrak{p}$. In fact, it is a well-known fact that

$$
\mathbb{C}[\mathfrak{p}]^{K}=\mathbb{C}\left[u_{1}, u_{2}\right],
$$

but we shall not use this here.

Lemma 2.2. The level sets for $u=\left(u_{1}, u_{2}\right)$ are single $K$-orbits.

Proof. Each $K$-orbit is uniquely determined by a set of eigenvalues (with multiplicities). It is easily seen that the characteristic polynomial of a trace free $3 \times 3$-matrix $X$ is

$$
-x^{3}+\frac{1}{2} u_{1}(X) x+u_{2}(X)
$$

The lemma follows immediately.

It follows that $W$ belongs to $\mathcal{X}$ if and only if it has a non-trivial intersection with each level set. Notice that $u_{1}(X)$ is the square of the trace norm of $X$, for $X$ symmetric. In particular, $u_{1}(X)>0$ for $X \neq 0$. Since $u_{1}$ and $u_{2}$ are homogeneous, it suffices to consider level sets of the form $\left\{u_{1}=1, u_{2}=c_{2}\right\}$.

We thus consider for each $W \in \operatorname{Gr}_{2}(\mathfrak{p})$ the unit sphere

$$
W_{1}=\left\{X \in W \mid u_{1}(X)=1\right\},
$$

and we define

$$
J:=\left\{u_{2}(X) \mid X \in W_{1}\right\} .
$$

Since $W_{1}$ is connected, $J$ is an interval. Moreover, it is symmetric around 0 , since $u_{2}$ has odd degree. In particular, we denote by

$$
I:=\left\{u_{2}(X) \mid X \in \mathfrak{a}_{1}\right\}
$$

the interval corresponding to the unit sphere in $\mathfrak{a}$. We now show:

Lemma 2.3. The interval $I$ is given by $I=[-c, c]$, where $c=54^{-1 / 2}$. Furthermore, the extreme values $\pm c$ are obtained precisely in those elements $X \in \mathfrak{a}_{1}$, which have two equal eigenvalues.

Proof. Let us introduce coordinates for $\mathfrak{a}$, namely

$$
\mathfrak{a}=\left\{D_{x y}:=\operatorname{diag}(x, y,-x-y) \mid x, y \in \mathbb{R}\right\} .
$$


Furthermore, let us introduce two functions:

$$
\begin{aligned}
& f_{1}(x, y):=u_{1}\left(D_{x y}\right)=2\left(x^{2}+y^{2}+x y\right) \\
& f_{2}(x, y):=u_{2}\left(D_{x y}\right)=-x y(x+y) .
\end{aligned}
$$

We wish to maximize/minimize $f_{2}$ under the condition of $f_{1}=1$. For that we perform the method of Lagrange: $d f_{1}=2(2 x+y, 2 y+x)$ and $d f_{2}=-(y(2 x+$ $y), x(2 y+x))$ have to be collinear. This can only happen in three cases: $x=y$, $2 x+y=0$ or $2 y+x=0$. Notice that these are exactly the cases in which two of the diagonal entries of $D_{x y}$ are equal.

We start with $x=y$. Here $f_{1}(x, x)=6 x^{2}=1$ means that $x= \pm 6^{-1 / 2}$. Hence $f_{2}(x, x)=-2 x^{3}= \pm 54^{-1 / 2}$. Secondly, if $2 x+y=0$, then $f_{1}(x,-2 x)=6 x^{2}=1$, so again $x= \pm 6^{-1 / 2}$. Hence $f_{2}(x,-2 x)=-2 x^{3}= \pm 54^{-1 / 2}$. Finally, the case $2 y+x=0$ is similar.

In order to complete the proof of Proposition 2.1, we only have to note that, as $\mathfrak{p}=K \cdot \mathfrak{a}$ we have $J \subset I$ and equality $J=I$ holds if and only if $W \in \mathcal{X}$.

Remark 2.4. (a) It follows from Proposition 2.1 that not all 2-dimensional subspaces $W \subset \mathfrak{p}$ belong to $\mathcal{X}$. An extreme case is

$$
W=\left\{\left(\begin{array}{ccc}
0 & 0 & 0 \\
0 & \lambda & \mu \\
0 & \mu & -\lambda
\end{array}\right) \mid \lambda, \mu \in \mathbb{R}\right\} .
$$

for which $u_{2}(W)=\{0\}$ and hence $W \notin \mathcal{X}$.

(b) Let us define a continuous function on $\mathrm{Gr}_{2}(\mathfrak{p})$ by

$$
f: \operatorname{Gr}_{2}(\mathfrak{p}) \rightarrow \mathbb{R}_{\geq 0}, \quad W \mapsto \max _{X \in W_{1}} u_{2}(X)
$$

Then we get

$$
\mathcal{X}=\left\{W \in \mathrm{Gr}_{2}(\mathfrak{p}) \mid f(W)=54^{-1 / 2}\right\}
$$

by our previous result. In particular, $\mathcal{X}$ is a closed subset of $\mathrm{Gr}_{2}(\mathfrak{p})$.

3. $K$-orbits on $\mathcal{X}$. We aim to describe $\mathcal{X}$ explicitly. Our starting point is the following observation. Let

$$
X_{0}:=\left(\begin{array}{ccc}
1 & 0 & 0 \\
0 & 1 & 0 \\
0 & 0 & -2
\end{array}\right),
$$

and observe that the two dimensional subspace $W \in \mathrm{Gr}_{2}(\mathfrak{p})$ belongs to $\mathcal{X}$ if and only if it contains a vector in the $K$-orbit of $X_{0}$.

In fact this is an immediate consequence of our discussion in the previous section. Since we consider trace free matrices, $X$ has two equal eigenvalues if and only if its eigenvalues are $\nu, \nu,-2 \nu$ for some $\nu \in \mathbb{R}$, that is, $X$ is conjugate to $\nu X_{0}$. 
Let $\Omega$ denote the 3 -dimensional variety

$$
\Omega=\left\{W \in \mathrm{Gr}_{2}(\mathfrak{p}) \mid X_{0} \in W\right\},
$$

in $\operatorname{Gr}_{2}(\mathfrak{p})$. The stabilizer $H \subset K$ of the line $\mathbb{R} X_{0}$ acts on $\Omega$. We have proved the following result:

Proposition 3.1. The map $(k, W) \mapsto k \cdot W$ from $K \times_{H} \Omega$ to $\mathcal{X}$ is surjective.

Notice that $H=M T$ where $T$ is the maximal torus

$$
T:=\left(\begin{array}{cc}
\mathrm{SO}(2, \mathbb{R}) & 0 \\
0 & 1
\end{array}\right)
$$

and $M \simeq[\mathbb{Z} / 2 Z]^{2}$ is the diagonal group group generated by

$$
m_{1}:=\left(\begin{array}{ccc}
-1 & 0 & 0 \\
0 & -1 & 0 \\
0 & 0 & 1
\end{array}\right), \quad m_{2}:=\left(\begin{array}{ccc}
1 & 0 & 0 \\
0 & -1 & 0 \\
0 & 0 & -1
\end{array}\right) .
$$

in $K$.

For $Y \notin \mathbb{R} X_{0}$ we set

$$
W_{Y}:=\operatorname{span}_{\mathbb{R}}\left\{X_{0}, Y\right\},
$$

then $\Omega=\left\{W_{Y} \mid Y \notin \mathbb{R} X_{0}\right\}$. Since $X_{0}$ is fixed under the maximal torus $T$, we have that $W_{Y}$ and $W_{t \cdot Y}$ belong to the same $K$-orbit for $t \in T$. Thus it suffices to consider elements $Y$ of the following shape:

$$
Y=Y_{\alpha, \delta, \epsilon}=\left(\begin{array}{ccc}
\alpha & 0 & \delta \\
0 & -\alpha & \epsilon \\
\delta & \epsilon & 0
\end{array}\right)
$$

Let $\mathcal{Y}$ denote the 2-dimensional projective space of these lines and consider the algebraic mapping

$$
K \times \mathcal{Y} \rightarrow \mathcal{X}, \quad(k,[Y]) \mapsto k \cdot W_{Y}
$$

The group $H$ does not act on $\mathcal{Y}$. However, let $N_{0}$ denote the subgroup of order 8 , generated by

$$
s_{0}=\left(\begin{array}{ccc}
0 & 1 & 0 \\
-1 & 0 & 0 \\
0 & 0 & 1
\end{array}\right)
$$

and $m_{2}$ (note that $\left.m_{1}=s_{0}^{2}\right)$. It follows from the relations

$$
s_{0} \cdot Y_{\alpha, \delta, \epsilon}=Y_{-\alpha,-\epsilon, \delta}, \quad m_{2} \cdot Y_{\alpha, \delta, \epsilon}=Y_{\alpha,-\delta, \epsilon} \cdot
$$

that $N_{0}$ acts on $\mathcal{Y}$. Conversely, if $k \in H$ and $k \cdot Y \in \mathcal{Y}$ for some $Y=Y_{\alpha, \delta, \epsilon} \in \mathcal{Y}$ with $\alpha \neq 0$, then $k \in N_{0}$.

Since furthermore $k \cdot W_{Y}=W_{k \cdot Y}$ for $Y \in \mathcal{Y}$ and $k \in N_{0}$, the above map (3.1) factorizes to an algebraic map

$$
K \times_{N_{0}} \mathcal{Y} \rightarrow \mathcal{X}
$$


We define the following open dense subset of $\mathcal{Y}$ :

$$
\mathcal{Y}^{\prime}=\left\{\left[Y_{\alpha, \delta, \epsilon}\right] \mid \alpha \neq 0, \delta \neq 0, \epsilon \neq 0\right\}
$$

and note that it is preserved by $N_{0}$.

Theorem 3.2. The algebraic map

$$
K \times_{N_{0}} \mathcal{Y} \rightarrow \mathcal{X}, \quad[k,[Y]] \mapsto k \cdot W_{Y}
$$

is $K$-equivariant, continuous and onto. Moreover, the restriction

$$
K \times_{N_{0}} \mathcal{Y}^{\prime} \rightarrow \mathcal{X}
$$

is injective. Its image $\mathcal{X}^{\prime}=\left\{k \cdot W_{Y} \mid k \in K,[Y] \in \mathcal{Y}^{\prime}\right\}$ is dense in $\mathcal{X}$ and carries a natural structure of a smooth 5-dimensional manifold.

The first statement was seen already. In order to obtain the statements about $\mathcal{X}^{\prime}$, we study the intersection of the $K$-orbit of $X_{0}$ with $W_{Y}$. We first prove:

Lemma 3.3. Assume

$$
X:=\left(\begin{array}{ccc}
\lambda & 0 & \delta \\
0 & \mu & \epsilon \\
\delta & \epsilon & -(\lambda+\mu)
\end{array}\right) \in K \cdot X_{0} .
$$

Then $\delta=0$ or $\epsilon=0$.

Proof. It follows from Lemma 2.2 that

$$
\begin{aligned}
& u_{1}(X)=2\left(\lambda^{2}+\mu^{2}+\lambda \mu+\delta^{2}+\epsilon^{2}\right)=u_{1}\left(X_{0}\right)=6 \\
& u_{2}(X)=-\lambda \mu(\lambda+\mu)-\epsilon^{2} \lambda-\delta^{2} \mu=u_{2}\left(X_{0}\right)=-2 .
\end{aligned}
$$

In particular, it follows from (3.4) that $\lambda^{2}+\mu^{2}+\lambda \mu \leq 3$. Since $\lambda^{2}+\mu^{2}+\lambda \mu=$ $\left(\lambda+\frac{1}{2} \mu\right)^{2}+\frac{3}{4} \mu^{2}$ this implies $|\mu| \leq 2$.

Multiplying by $\frac{1}{2} \mu$ in (3.4) and adding (3.5) we obtain

$$
\mu^{3}+\epsilon^{2} \mu-\epsilon^{2} \lambda=3 \mu-2,
$$

or equivalently

$$
\epsilon^{2}(\lambda-\mu)=(\mu+2)(\mu-1)^{2} .
$$

In particular it follows that $\epsilon=0$ or $\lambda \geq \mu$.

Since $\mu$ and $\lambda$ appear symmetrically in (3.4) and (3.5) we obtain similarly $|\lambda| \leq 2$

$$
\delta^{2}(\mu-\lambda)=(\lambda+2)(\lambda-1)^{2},
$$

and conclude that $\delta=0$ or $\mu \geq \lambda$.

Notice finally that if $\lambda=\mu$, then $\lambda=\mu=-2$ or $\lambda=\mu=1$ by (3.6), and from (3.4) it then follows that $\lambda=\mu=1$ and $\delta=\epsilon=0$.

We can now prove Theorem 3.2. 
Proof. It remains to be seen that $k \cdot W_{Y}=W_{Y^{\prime}}$ implies $k \in N_{0}$ and $\left[Y^{\prime}\right]=[k \cdot Y]$ for $Y, Y^{\prime} \in \mathcal{Y}^{\prime}$. In particular, it follows from $k \cdot W_{Y}=W_{Y^{\prime}}$ that $k \cdot X_{0}=a X_{0}+b Y^{\prime}$ for some $a, b \in \mathbb{R}$. Since $Y^{\prime} \in \mathcal{Y}^{\prime}$ it follows from Lemma 3.3 that $b=0$, and hence $k \in H$. Now $k \cdot Y$ must be a multiple of $Y^{\prime}$, by orthogonality with $X_{0}$ with respect to the trace form $\langle A, B\rangle=\operatorname{tr}(A B)$. It follows that $k \in N_{0}$.

In order to give a complete classification of $\mathcal{X}$, one needs to describe the fibers in $K \times \mathcal{Y}$ above the elements outside of $\mathcal{X}^{\prime}$. We omit the details, but mention that in general the fibers will not be finite.

\section{References}

[1] A. W. KNAPP, Lie groups beyond an introduction, Birkhäuser, 2002.

Bernhard Krötz, Max-Planck-Institut für Mathematik, Vivatsgasse 7, D-53111 Bonn, Germany

e-mail: kroetz@mpim-bonn.mpg.de

Henrik SchlichtKrull, Department of Mathematical Sciences, University of Copenhagen, Universitetsparken 5, DK-2100 Copenhagen, Denmark

e-mail: schlicht@math.ku.dk

Received: 27 June 2008 